Ann. Biol. anim. Bioch. Biophys., I967, 7 (I), 73-78.

\title{
ÉTUDE DE LA TRANSFERRINE DES HYBRIDES INTERSPECIFIQUES GALLUS GALLUS × NUMIDA MELEAGRIS
}

\author{
G. CROIZIER \\ avec la collaboration technique de J.-P. HARSCOAT \\ Station de Recherches avicoles, \\ Centre national de Recherches zootechniques, 78-Jouy-en-Josas
}

\section{SOMMAIRE}

Chez l'hybride Poule $\times$ Pintade nous avons mis en évidence, par électrophorèse en gel d'amidon, des variations individuelles de la transferrine sérique.

Après analyse électrophorétique du sérum de poule et du sérum de pintade, il nous a été possible d'établir une corrélation entre le polymorphisme génétique existant chez l'hybride et celui déjà connu chez la poule. Les hybrides étudiés ont hérité de l'allèle $\mathrm{T}^{\mathbf{A}}$ ou de l'allèle $\mathrm{T} f^{\mathbf{B}}$ de transferrine selon le génotype du Gallus parent.

L'électrophorèse permet de déceler une hétérogénéité moléculaire de la transferrine sérique aussi bien chez les sujets hybrides que chez les individus des espèces parentales. Cette technique révèle la présence de deux populations de molécules dans la transferrine de poule et de trois popu. lations de molécules dans la transferrine de pintade. Le type le plus lent de molécule de la transferrine de pintade ne figure plus dans le sérum de l'hybride.

\section{INTRODUCTION}

Il est rare de pouvoir suivre le passage et le devenir des gènes parentaux dans les hybrides interspécifiques. BECKMAN, Contario et MAINARDI (I963) trouvent dans le sérum des hybrides interspécifiques d'oiseaux les sérumalbumines des deux parents à l'exclusion de molécules hybrides. QuINTFros et al. (I964) font à partir d'hybrides fertiles de Dindons (Meleagris gallopavo par Meleagris ocellata) une observation analogue. Ces auteurs attribuent le polymorphisme de la serumalbumine rencontré, chez les descendants de ces hybrides, à deux allèles codominants prove- 
nant respectivement des deux espèces parentales. HALEY (I965) observe qu'une caille hétérozygote pour la sérumalbumine transmet à chacun de ses descendants, soit un allèle, soit 1'autre après croisement avec Gallus gallus L.

Le travail présenté étend à une deuxième protéine sérique, la transferrine, les résultats obtenus à partir de la sérumalbumine.

Il nous a été possible de trouver chez des hybrides de Gallus gallus L. par Numida meleagris un polymorphisme de la transferrine et de 1'homologuer à celui décrit chez la poule par OGDEN et al. (I962).

\section{I. -- MA'TÉRIEL ET MÉTHODES}

\section{Matériel animal}

Les hybrides Poule $\times$ Pintade ont été obtenus par Petitjean ( $\left.{ }^{1}\right)$. Ils se composent de 8 sujets âgés de trois ans et de II 5 sujets âgés de trois mois. Le sens du croisement (coq Gallus domesticus $\times$ poule Numida meleagris) est le même pour tous les animaux sauf pour un hybride de trois ans qui provient du croisement inverse.

Les pintades appartiennent à la variété gris perle commune. Pour les hybrides de trois ans les coqs parents sont de type Cornish et pour les hybrides de trois mois de souche Rhode Island Red. La seule poule mère d'un hybride est une Rhode Island Red.

Le sang est prélevé dans la veine alaire à l'aide d'une seringue.

\section{Méthodes}

Les transferrines de la poule, de la pintade et des hybrides ont été étudiées et comparées dans les mêmes conditions expérimentales par électrophorèse en gel d'amidon.

Après séparation électrophorétique des protéines sériques, la transferrine a été révélée et identifiée par autoradiographie $\left({ }^{59} \mathrm{Fe}\right)$ puis révélée simplement, par la suite, par un colorant général des protéines.

\section{Électrophorèse.}

\section{Techniques}

L'électrophorèse de zone en gel d'amidon de KRISTJANsson (1963) a été employée.

La durée de l'électrophorèse est de trois heures. Le front de migration se situe alors à II $\mathrm{cm}$ de la ligne d'insertion. Dans ces conditions la sérumalbumine n'est pas détachée du front de migration. Les bandes de transferrine, la plus rapide et la plus lente sont écartées de $3,5 \mathrm{~cm}$; la plus rapide de ces bandes est située à une distance de $8 \mathrm{~cm}$ à partir de la ligne de départ.

\section{Autoradiographie.}

Avant l'électrophorèse le sérum est incubé pendant $3 \circ$ minutes à $37^{\circ} \mathrm{C}$ avec un volume égal de solution de ${ }^{58} \mathrm{Fe}$ (acétate). L'activité finale du mélange est de $10 \mu \mathrm{C} / \mathrm{ml}$.

Après l'électrophorèse le gel de $6 \mathrm{~mm}$ d'épaisseur est partagé en deux parties. La partie inférieure, de $2 \mathrm{~mm}$ d'épaisseur, est utilisée pour la révélation autoradiographique. La partie supérieure, de $4 \mathrm{~mm}$ d'épaisseur, sert à la coloration des protéines et permet d'établir une correspondance entre ces deux révélations de la transferrine. La superposition des bandes de transferrine obtenue pour ces deux révélations localise cette protéine sur l'électrophorégramme.

Un film Régulix Kodak est appliqué sur la tranche inférieure du gel, une feuille mince en plastique est intercalée entre le film et le gel. L'exposition a lieu en chambre à $-20^{\circ} \mathrm{C}$ et dure 72 heures. Nous avons utilisé le Révélateur L X 24 et le Fixateur A L 4 employés par Kodak pour révéler les films Régulix.

(1) Station expérimentale avicole du Magneraud. 


\section{II. - RÉSULTATS}

Transferrine de la Poule.

Ire polymorphisme de la transferrine impliqué dans 1'étude présente a été décrit initialement par OGDEN et al. (I962) puis par WILLIAMs (I962). L'étude de la transferrine de la poule fait l'objet d'une autre publication (CroIzIER, I967). Les phénotypes $T f^{\wedge}$ et $T f^{B}$ de transferrine de la poule, tels qu'ils apparaissent dans nos conditions d'électrophorèse, sont représentés dans le schéma $\mathrm{I}$ (colonnes 2 et 5 ) et peuvent être observés sur la photographie r. Ainsi chacun de ces deux variants génétiques de la transferrine (variant $A$ et variant $B$ ) s'extériorise sous la forme de deux bandes. Le variant $A$ possède la vitesse de migration électrophorétique la plus grande.
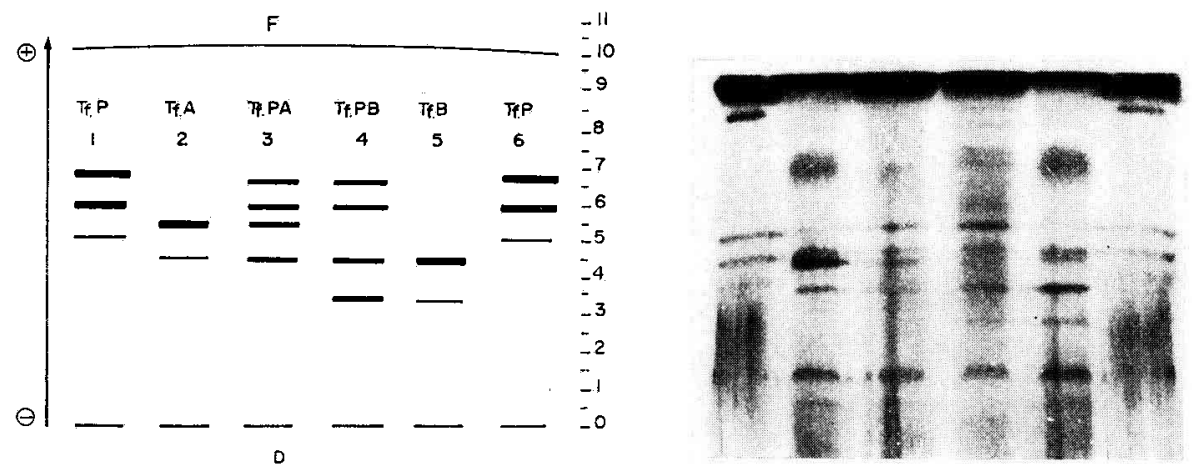

FIG. I. - Comparaison des phénotypes de transterrines de Pintade, de Poule et d'hybride Poule $\times$ Pintade.

Colonnes $\mathrm{I}$ et 6 : Pintade $\mathrm{T} f^{\mathrm{P}}$

Colonnes 2 et 5 : Poules $\mathrm{T} / \mathrm{A}$ et $\mathrm{T} / \mathrm{B}$

Colonnes 3 et $4:$ Hybrides $\mathrm{T} / \mathbf{P A}$ et $\mathrm{T} / \mathbf{P B}$

Transferrine de la Pintade.

Ellle donne lieu en électrophorèse à la formation de trois bandes révélées d'une manière identique par autoradiographie et par coloration générale des protéines : figure I, (colonnes I et 6).

Aucune différence individuelle n'a été perçue à partir du sérum des 54 pintades femelles reproductrices mères des II 5 hybrides.

La transferrine de la pintade possède une vitesse de migration électrophorétique supérieure à celle de la transferrine de poule.

Comme il est possible de le constater à partir đu schéma I et de la photographie $\mathrm{I}$, la bande la plus rapide 'de la transferrine de poule (de génotype $T f^{\Delta / T} /{ }^{\Delta}$ ) est située un peu en avant de la bande la plus lente de la transferrine de la pintade. De plus il faut noter que la bande la plus lente de la transferrine de pintade est beaucoup moins marquée que les deux autres. 
Transferrine de l'hybride.

Nous avons observé deux phénotypes de transferrine. Pour II 7 sérums sur I23 la transferrine s'extériorise, après l'autoradiographie ou la coloration générale des protéines, sous la forme de quatre bandes, deux bandes sont situées à la hauteur des deux bandes principales de la transferrine de pintade et les deux autres bandes migrent comme les deux bandes de transferrine du variant B de la poule. Pour les 6 autres sérums, des quatre bandes présentes, deux migrent à la même vitesse que celle des deux bandes principales de la transferrine de pintade et les deux autres bandes, elles, migrent comme les deux bandes du variant $A$ de la transferrine de poule. Pour la comparaison de la transferrine de poule, de pintade et d'hybride se reporter au schéma I et à la photographie $I$.

Les II5 hybrides analysés à l'âge de trois mois, proviennent d'une reproduction pedigree à laquelle participent effectivement 22 coqs Rhode Island Red de génotype $\mathrm{T} f^{\mathrm{B}} / \mathrm{T} f^{\mathrm{B}}$ et 54 pintades auxquelles par analogie on peut attribuer le génotype $\mathrm{T} f^{\mathrm{P}} / \mathrm{T} f^{\mathrm{P}}$. Tous ces oiseaux possèdent le même phénotype de transferrine, celui que nous avons précédemment décrit en premier. Nous attribuons à ce phénotype le symbole T $f$. PB $\mathrm{T} f^{\mathrm{pB}}$.

Les 8 hybrides étudiés à l'âge de trois ans extériorisent les deux phénotypes décrits. Il ne nous a pas été possible d'analyser le sérum des parents. I'unique hybride Numida meleagris $\times$ Gallus gallus a une mère Rhode Island Red et présente le phénotype Tf. PB T $f^{\mathrm{PB}}$. Pour les 7 autres hybrides tout se passe comme si 6 d'entre eux avaient reçu de leur père (coq Cornish) l'allèle $T f^{\wedge}$ et le $7^{\mathrm{e}} 1^{\prime}$ 'allèle $\mathrm{T} f^{\mathrm{B}}$. Nous attribuons le symbole $\mathrm{T} f$. PA. T $\mathrm{P}^{\mathrm{A} A}$ au phénotype commun aux 6 hybrides, le $7^{\mathrm{e}}$ hybride est de phéno-type $\mathrm{T} f$. PB T $f^{\mathrm{PB}}$.

\section{DISCUSSION ETT CONCLUSIONS}

L'étude par électrophorèse de la transferrine de diverses espèces animales met en évidence des polymorphismes de deux natures. Le premier type de polymorphisme est sous le contrôle d'une série allélique au locus T $f$; il concerne la population animale : les différents gènes de la série allélique $\mathrm{T} f$ modifient la vitesse de migration électrophorétique de la transferrine sérique. L'hétérogénéité électrophorétique de la transferrine sérique constitue le deuxième type de polymorphisme; il concerne l'espèce.

Le nombre des allèles situés au locus $\mathrm{T} f$ peut être très différent che $z$ deux espèces phylogénétiquement très proches. Le genre Maccaca en fournit un exemple (GooDMAN et PouLiK, I96r). La Poule et la Pintade appartiennent à deux genres différents. Seule la poule possède un polymorphisme de cette nature (OGDEN et al., I962; Stratil, I966; Croizier I966 et I967). Il faut cependant remarquer que la Pintade n'a fait l'objet que d'un nombre d'études très restreint.

Un même allèle de transferrine peut contrôler la synthèse de plusieurs espèces moléculaires (PARKER et BEARN, I963). Ainsi dans les conditions expérimentales que nous avons employées, la transferrine sérique de poule présente deux populations de molécules et celle de pintade en présente trois. Les mécanismes qui sont à 1'origine 
de ces diverses populations moléculaires sont mal connus. YoshiokA, FUJII et ITo (Ig66) ont obtenu la séparation électrophorétique de la transferrine libre et de la transferrine liée au fer. La concordance qui existe, dans notre expérience, entre les résultats obtenus par autoradiographie et par coloration montre que le fer fixé aux molécules de transferrine n'affecte pas leur vitesse de migration électrophorétique. Ce problème est abordé dans une autre publication (CROIZIER, I967).

Il ne nous a pas été possible de retrouver dans le sérum de 1'hybride Gallus $\times$ Numida l'espèce moléculaire la plus lente de la transferrine de Numida. On peut en déduire soit que cette fraction est absente du sérum, soit qu'elle y est en quantité insuffisante pour être détectée par les techniques utilisées.

La première hypothèse est rendue plausible par les constatations de WILLIAMS (I962) et de PARKER et BEARN (I963) selon lesquelles la synthèse de formes différentes de transferrine peut se produire au niveau des tissus distincts. La transferrine sérique serait synthétisée par le foie et l'ovotransferrine ou conalbumine serait, elle, synthétisée au niveau de l'oviducte. La cellule de l'hybride constitue par rapport aux cellules parentales qui produisent la transferrine un milieu de synthèse particulier.

En faveur de la deuxième explication il faut noter que la bande la plus lente de transferrine de la Pintade est relativement très peu marquée. Cependant si l'absence de cette bande dans le phénotype de l'hybride ne permet pas de conclure à la disparition de la protéïne sérique correspondante, on est au moins conduit à admettre une diminution marquée de la synthèse de cette fraction de transferrine. Cette diminution paraît plus importante que celle que l'on doit prévoir du fait de la présence d'un seul gène parental.

\section{Conclusions}

L'étude de la transferrine sérique chez l'hybride Gallus gallus L. $\times$ Numida meleagris montre que cet oiseau hérite un gène de structure de chacun de ses parents.

Ce résultat est en accord avec celui obtenu chez d'autres hybrides par l'étude de la sérumalbumine.

L'absence, ou la réduction, de la synthèse d'une fraction de la transferrine de Pintade chez l'hybride constitue un nouvel exemple de l'influence du lieu de formation d'une protéine sur sa composition.

Rę̧ pour publication en janvier I967.

\section{REMERCIEMENTS}

Ce travail a pu être réalisé grâce à l'aide de plusieurs personnes que nous sommes heureux de remercier :

- P. Nizza du Commisariat à l'Énergie atomique, nous a fourni le fer radioactif. En matière d'utilisation des isotopes radioactifs nous avons bénéficié de la grande compétence de L. GUEGEN, Laboratoire des Métabolismes Centre national de Recherches zootechniques.

- M. Petitjean, Station expérimentale avicole du Magneraud nous a procuré les échantillons de sang indispensables à cette étude. 


\title{
SUMMARY
}

\author{
A STUDY OF TRANSFERRIN IN INTERSPECIFIC \\ "GALLUS GALLUS " $X$ " NUMIDA MELEAGRIS" HYBRIDS
}

Starch-gel electrophoresis (KRISTJANSSON, I963) allowed us to notice two variants of serum transferrin in the Gallus Gallus $\mathrm{L} \times$ Numida mel agris hybrid. Transferrin was traced by ${ }^{59} \mathrm{Fe}$ autoradiography.

Electrophoresis allows to point out a molecular heterogeneity of serum transferrin in hybrids as well as in each species. This technique reveals the presence of two populations of molecules in the hen's transferrin and three populations in the Guinea-Fowl.

After an electrophoretic analysis of the hen's and Guinea-Fowl's serum, a correlation could be established between the genetic polymorphisms of the hybrid and of the hen. The investigated hybrids have inherited the $\mathrm{T} f$. A or $\mathrm{T} f$. B allele of transferrin according to the genotype of the parent Gallus. Figure I entitles a comparison between Guinea-Fowl transferrin (Tf. P), Hen transferrin (T $f$. A or T $f . \mathrm{B}$ ), and hybrid transferrin (Tf. $\mathrm{PA}$ or $\mathrm{T} f . \mathrm{PB})$. I 17 hybrids out of $\mathrm{I} 23$ have the $\mathrm{T} f$. $\mathrm{PB}$ variant, 6 the $\mathrm{T} f$. PA. Therefore :

- The study of serum transferrin in the Gallus Gallus $\mathrm{L} \times$ Numida meleagris hybrid shows that this bird inherits a structure gene from each parent.

- The slowest type of Numida transferrin molecule is no more present in the hybrid's serum. This absence, or at least lower rate, of synthesis of a fraction of Numida transferrin in the hybrid is another instance of the influence that the place where a protein is formed can have on its composition.

\section{RÉFÉRENCES BIBLIOGRAPHIQUES}

Beckman I., Conterio F., Mainardi D., ig63. Serum protein variations in bird species and hybrids. The Serological museum Bull., 29, 5-8.

Croizier G., ig66. Polymorphismes biochimiques de la poule domestique. I. Analyse génétique des protéines du blanc d'œuf chez des poules de races françaises et étrangères. Ann. Biol. anim. Biochim. Biophys., 6, $379-388$.

Croizier G., 1967. Polymorphismes biochimiques de la poule domestique. II. Contrôle génétique du polymorphisme de la transferrine. Ann. Biol. anim. Biochim. Biophys. (sous presse).

Goodman M., Poulik E., I96r. Serum transferrins in the genus Maccaca. Nature, 191, I407-I408.

HALEY L. E., I965. Serum albumin polymorphism in quail and chicken-quail hybrids. Genetics. 51,983986.

Kristjansson F. K., I963. Genetic control of two pre-albumins in pigs. Genetics, 48, 1059-1063.

Ogden A. L., Morton J., Gilmour D., McDermid, ig6z. Inherited variants in the transferrins and conalbumins of chicken. Nature, 195, 1026-1 028.

Parker W. C., Bearn A. G., 1963. Boric acid induced heterogeneity of conalbumin by starch-gel electrophoresis. Nalure, 199, г I $84-$ I 186.

Quinteros I. R., 1964. Albumin phenotypes in turkeys. Genetics, 50, 579-582.

STRATIL A., I966. A contribution towards the study of transferrins and conalbumins of the domestic fowl. In Congrès des groupes sanguins des animaux. Paris. (sous presse).

Williams J., I962. A comparison of conalbumin and transferrin in the domestic fowl. Biochem. J., 83, $355-364$.

Yoshioka R., FujII J., Iтo Y., . 1966. On electrophoretic resolution and densitometric determination of Apo-Transferrin and iron bound transferrin. Biochem. biophys. Res. Commun., 24, 203-207. 